\title{
Clinical significance of C4d deposition in renal tissues from patients with primary Sjögren's syndrome-a preliminary study
}

\author{
Wenli Xia ${ }^{1,2}$, Bixia Gao ${ }^{3}$, Lin Duan', Yan Li', Yubing Wen', Limeng Chen', Xuemei Li ${ }^{1}$, Falei Zheng ${ }^{1}$ and
} Mingxi $\mathrm{Li}^{1 *} \mathbb{D}$

\begin{abstract}
Background: To evaluate renal expression of $\mathrm{C} 4 \mathrm{~d}$, a complement component in the classical/mannose binding lectin (MBL) pathway, in patients with primary Sjögren's syndrome (pSS)-associated renal impairments.

Methods: We retrospectively reviewed the clinical and pathological data from 39 patients with pSS presenting with renal impairments. C4d was examined in paraffin-embedded biopsy tissues using immunohistochemistry. Glomerular C4d positive was defined when $>75 \%$ glomeruli were globally stained. Tubulointerstitial C4d (TI-C4d) were scored semi-quantitatively as 0 (absent), 1 (spotty or weak), 2 (patchy) and 3 (diffuse). A TI-C4d score $\geq 2$ was considered TIC4d positive and included in the TI-C $4 \mathrm{~d}^{+}$group and vice versa. Peritubular capillary (PTC) C4d was scored as 0 (absent), 1 (0 10\%, minimal), 2 (10\% 50\%, focal), and 3 (> 50\%, diffuse).
\end{abstract}

Results: Glomerular C4d deposition was observed in all 8 patients with pSS-related membranous nephropathy (MN) without obvious $\mathrm{C} 1 \mathrm{q}$ deposition. Two of 5 patients with mesangial proliferative glomerulonephritis and 1 of 2 patients with IgA nephropathy had mild mesangial C4d deposition. Sixteen patients ( 6 glomerular dominant and 10 tubulointerstitial dominant) presented TI-C $4 \mathrm{~d}$ score $\geq 2$. Patients in the $\mathrm{TI}-\mathrm{C}_{4} \mathrm{~d}^{+}$group exhibited a higher serum creatinine level at the time of renal biopsy $\left(\mathrm{TII}^{-C} 4 \mathrm{~d}^{+} 132.5[89.7,165.5] \mathrm{vs}\right.$. TI-C $\left.4 \mathrm{~d}^{-} 83.0[70.7,102.0] \mu \mathrm{mol} / \mathrm{L}, P=0.008\right)$. PTC C4d was observed in 12 patients, with each of minimal, focal and diffuse staining being noted in 4 patients.

Conclusions: The MBL pathway of complement activation was potentially involved in pSS-related MN. Tubulointerstitial C4d might be a pathological marker of severe renal injury in patients with pSS-related renal impairments.

Keywords: Primary Sjögren's syndrome, Renal involvement, C4d deposition

\section{Background}

The kidney is affected in approximately 0.3 to $33.5 \%$ of patients with primary Sjögren's syndrome (pSS), a systemic disease characterized by sicca symptoms and anti-Ro/SSA and/or anti-La/SSB antibodies [1-3]. Two main pathological entities have been described. Tubular interstitial nephritis (TIN) is the major type and is often characterized by renal tubular acidosis (RTA) with or without renal insufficiency [4]. Glomerulonephritis (GMN) is the other type, including membranoproliferative glomerulonephritis

\footnotetext{
* Correspondence: mingxili@hotmail.com

'Department of Nephrology, Peking Union Medical College Hospital, Chinese Academy of Medical Sciences, Peking Union Medical College, No. 1

Shuaifuyuan, Wangfujing Street, Beijing 100730, China

Full list of author information is available at the end of the article
}

(MPGN), membranous nephropathy (MN), mesangial proliferative glomerulonephritis (MePGN), focal segmental glomerular sclerosis (FSGS) and minimal change disease (MCD) [5-7]. MN (pSS-MN) is one of the major types of GMN reported in different studies $[3,8]$.

Primary membranous nephropathy (PMN) is a kidneyspecific disease with increased proteinuria and pathological characteristics of sub-epithelial immune complex deposition (predominantly IgG4 and C3 deposition) [9]. PMN is characterized by the presence of serum autoantibodies against the podocyte component M-type phospholipase A2 receptor (PLA2R) in approximately $70 \%$ of patients [10] and anti-thrombospondin type 1 domain containing 7A (THSD7A) antibodies in approximately $10 \%$ of those with negative anti-PLA2R antibodies (nearly $3 \%$ of patients with

(c) The Author(s). 2019 Open Access This article is distributed under the terms of the Creative Commons Attribution 4.0 International License (http://creativecommons.org/licenses/by/4.0/), which permits unrestricted use, distribution, and 
intrinsic antibodies related $\mathrm{MN}$ ) [11, 12]. The mannosebinding lectin pathway of complement activation is involved in PMN, as evidenced by sub-epithelial deposition of hypogalactosylated IgG4 and C3 and 100\% glomerular C4d deposition without C1q co-deposition [13].

Notably, pSS is one of the possible causes of secondary $\mathrm{MN}$ [3]. Patients with pSS-MN may present extra renal manifestations that do not exist in patients with PMN [6, 14]. In patients with $\mathrm{MN}$ secondary to pSS, the complement activation pathway has not been studied. Patients with pSS-TIN are characterized by interstitial lymphocyte infiltration with rare tubular and interstitial immune complex deposition [15]. Autoantibodies against tubular components have been identified in patients with pSS-TIN by some authors [16-18], and the complement component C9 was also observed around TBM in some patients with pSS-TIN [4]. It has not extensively researched whether immune complex-mediated interstitial injury plays a role in pSS-TIN.

C4d deposition in various compartments of renal tissues have been reported. C4d deposition in the glomerulus indicates local complement activation through the classical or lectin pathway [19]. It is used to differentiate C3 glomerulopathy and immune complex-mediated proliferative GN [20]. Mesangial C4d deposits have been observed in patients with IgA nephropathy (IgAN) [21], lupus nephritis (LN) [22], and thrombotic microangiopathy (TMA) [23]. Peritubular capillary (PTC) C4d correlates with antibody-mediated transplant rejection and inferior renal allograft outcomes [24]. Tubular C4d deposition is associated with an increased WHO grade of IgAN [25]. To our knowledge, neither glomerular C4d deposition nor tubulointerstitial C4d deposition have been investigated in patients with pSS-MN and in patients with pSS-TIN.
In the present study, we performed a retrospective examination of 39 patients with pSS with renal involvement (21 cases with TIN and 18 cases with GMN) from a single centre. C4d were examined in formaldehydefixed, paraffin-embedded biopsy tissues using immunohistochemistry. The objectives were to investigate the prevalence and localization of C4d deposits in renal biopsy tissues in patients with pSS-MN and those with pSS-TIN.

\section{Methods}

\section{Patient selection}

We retrospectively analysed the clinical and pathological data from 82 hospitalized patients with biopsy-proven renal involvements secondary to "Sjögren's syndrome" (ICD: M35.001) in Peking Union Medical College Hospital (PUMCH) from 1996 to 2011. After Sjögren's syndrome was redefined based on the 2002 American and European Classification Criteria (AE), we excluded 13 patients with systemic lupus nephritis (LN), 2 patients with primary biliary cirrhosis (PBC), 3 patients with rheumatoid arthritis (RA), 2 patients who were positive for anti-HCV antibodies, 2 patients with unidentified GMN due to glomerular sclerosis, and 3 patients for whom adequate kidney biopsy samples were unavailable. In addition, 18 patients who only qualified based on the Fox criteria which does not require the presence of anti-SSA/SSB antibodies were excluded. Thirty-nine patients were enrolled (Fig. 1). All inpatients received a consultation with a rheumatologist and had a confirmed diagnosis of primary Sjögren's syndrome. After patient selection, archived formalin-fixed and paraffin-embedded kidney tissues were retrieved and stained as described below. The study was performed in

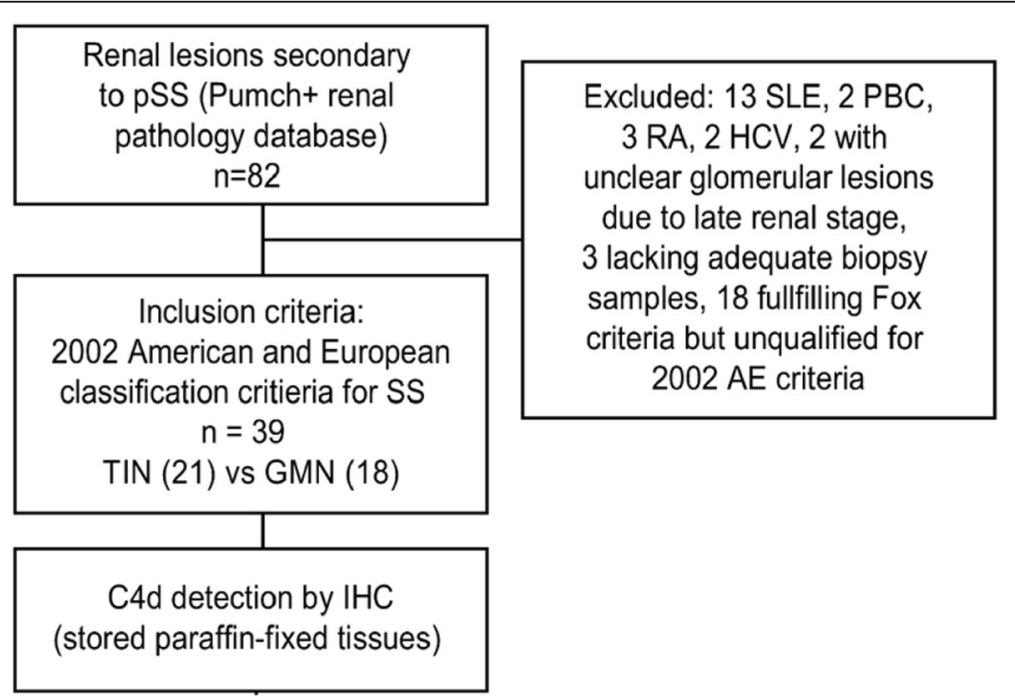

Fig. 1 Patient selection chart 
accordance with the ethical standards of the Declaration of Helsinki and was approved by the Institutional Review Board of PUMCH (Reference code:S-k585).

\section{Clinical data}

Medical records including the demographic data, the duration of pSS, the duration of renal impairment from symptom onset to renal biopsy, medical comorbidities and treatment history were retrospectively reviewed. The immunological screening for antinuclear antibodies (ANA), anti-SSA/SSB antibodies, immunoglobulins (IgG, IgM and IgA), complement (CH50, C3 and C4), C-reactive protein (CRP), rheumatoid factor (RF) and cryoglobulins were obtained.

Renal function was evaluated by the estimated glomerular filtration rate (eGFR) with the CKD Epidemiology Collaboration (CKD-EPI) equation. Renal tubular function screening included blood and urine $\mathrm{pH}$ values, electrolytes, blood and urine $\alpha 1$-macroglobulin and $\beta 2$-microglobulin level, urine transferrin protein and $\mathrm{N}$-acetyl- $\beta$-amino-glucosidase levels. Ammonium chloride load test and sodium hydrogen carbonate reabsorption rate were applied to verify the diagnosis of renal tubular acidosis. Urine and blood osmolality, fluid deprivation/vasopressin test and cranial images were evaluated to assess the renal tubular concentration function and to differentiate diabetes insipidus. Proteinuria based on 24-h urine protein excretion rate was classified as mild ( $<1.5 \mathrm{~g} /$ day), moderate $(1.5-3.5$ $\mathrm{g} /$ day) and nephrotic ( $>3.5 \mathrm{~g} /$ day). Urinary ultrasound results were reviewed for evidence of anomalies and renal calcification.

\section{Kidney pathology and C4d detection \\ Pathological examination of kidney tissue}

The renal histological diagnosis was based on light microscopy (LM), immunofluorescence (IF) staining and electron microscopy (EM) findings. The diagnosis was made by two independent pathologists. Patients were divided into the TIN group (with TIN only) and GMN group (including patients with interstitial lesions) based on pathological findings.

Kidney biopsy specimens were scored semi-quantitatively: (1) mesangial proliferation: 0 (no proliferation, $\leq 3$ mesangial cells per mesangial area), 1 (mild to moderate proliferation, 4-6 mesangial cells per area) and 2 (intense proliferation, $>6$ mesangial cells per area). (2) Glomerular sclerosis and crescent were counted as a percentage. Eight cases with no more than 15 glomeruli per specimen were only excluded from the calculation of this item. (3) Tubular atrophy and interstitial fibrosis were scored according to the percent area involved: 0 (absent), $1(\leq 25 \%), 2$
(25-50\%), 3 (50-75\%), and 4 (>75\%). IgG, IgM, IgA, $\mathrm{C} 1 \mathrm{q}, \mathrm{C} 3, \mathrm{C} 4$, fibrinogen, and light chain $\mathrm{\kappa}$ and $\lambda$ levels were detected using direct IF staining and scored as 0 (negative), $1(1+), 2(2+), 3(3+)$ and $4(4+)$. IF-positive samples were defined as positive for IgG, IgM, IgA, $\mathrm{C} 1 \mathrm{q}, \mathrm{C} 3, \mathrm{C} 4$, $\mathrm{k}$ or $\lambda$ and scored as $\geq 1$ point.

\section{Immunohistochemical staining of C4d}

Rabbit anti-human polyclonal C4d antibodies (1:40; Biomedica, Vienna, Austria) were applied on $2-\mu \mathrm{m}$ formaldehyde-fixed kidney sections. Antigen retrieval was performed by incubating the sections with $0.4 \%$ pepsin (Zhongshan Golden Bridge Biotechnology, Beijing, China) for $30 \mathrm{~min}$ at $37^{\circ} \mathrm{C}$. Sections were incubated with the primary antibody overnight at $4{ }^{\circ} \mathrm{C}$ and with avidin-free horseradish peroxidase (HRP)-conjugated goat anti-rabbit immunoglobulins using the two-step Envision kit (PV9001, Zhongshan Golden Bridge Biotechnology, Beijing, China) for $30 \mathrm{~min}$ at $37^{\circ}$ C. Sections were treated with a freshly prepared 3-3-diaminobenzidine solution (DAB) (Zhongshan Golden Bridge Biotechnology, Beijing, China) for $3 \mathrm{~min}$ and counter-stained with haematoxylin for $30 \mathrm{~s}$.

A positive control for C4d was obtained from dendritic lymphocytes in human tonsil tissues from a patient undergoing an operation for obstructive sleep apnoea hypopnea syndrome (Additional file 1: Figure S1) [26]. Renal tissues from patients with mild lesions who underwent a biopsy for microscopic haematuria were used as negative controls (Additional file 1: Figure S2).

\section{C4d evaluation and scoring in different compartments}

Glomerular C4d positive $\left(\mathrm{G}-\mathrm{C} 4 \mathrm{~d}^{+}\right)$was defined when $>75 \%$ glomeruli had global staining (involving $>50 \%$ area of one glomerulus), vice versa glomerular negative $\left(\mathrm{G}-\mathrm{C} 4 \mathrm{~d}^{-}\right)$[21].

Tubulointerstitial C4d (TI-C4d) deposition was semiquantitatively scored as 0 (absent), 1 (weak or spotty stain), 2 (patchy stain), and 3 (diffuse stain) within microscopic fields. A TI-C4d score $>1$ was defined as positive (TI-C4d $\left.\mathrm{d}^{+}\right)$ [27]. Using a TI-C4d deposit score of $>1$ as a cut-off, patients were classified into the $\mathrm{TI}-\mathrm{C} 4 \mathrm{~d}^{+} \mathrm{TI}-\mathrm{C} 4 \mathrm{~d}^{-}$groups.

Peritubular capillary C4d (PTC-C4d) staining was evaluated and graded according to the Banff criteria as negative $(0$, absent), minimal ( 1 , less than $10 \%)$, focal (2, between 10 and $50 \%)$ and diffuse $(3,>50 \%)$ [28].

\section{Statistics}

Continuous variables with a normal distribution were analysed using Student's t-test or the Mann-Whitney U-test for abnormally distributed data. Categorical data were compared using the Fisher's exact test. Correlations were analysed by calculating Pearson's correlation coefficients for continuous normally distributed 
variables, Spearman's Rho for nominal and ordinal variables, and performing a linear regression analysis for continuous variables. $P<0.05$ was considered statistically significant. Calculations were performed using SPSS statistical software version 11.5 (SPSS, Chicago, IL, USA).

\section{Results}

\section{Demographic characteristics of patients with pSS} presenting renal involvement

Among the 39 patients included in this study, 21 were diagnosed with TIN, and 18 were diagnosed with GMN. Eight of 18 patients with GMN also had TIN. The male:female ratio was 9:30. The average age of the patients was $42 \pm 14.1(9-72)$ years at the time of renal biopsy. ANA was positive in $37 / 39$ patients, with different titres: 8 patients at a 1:1280 titre, 8 patients at 1:640, 9 patients at 1:320, 9 patients at $1: 160,2$ patients at 1:80, and 1 patient at 1:40.
Hyper- $\gamma$-globulinemia was present in 11/36 (28.2\%) patients. Elevated serum Ig levels and hypocomplementemia were also observed (Table 1). Twenty-two (56.4\%) patients were diagnosed with RTA (18 type I cases and 4 type II cases). Three of the 4 patients with type II RTA simultaneously exhibited Fanconi syndrome. Twenty (48.8\%) patients had polynocturia, and two had diabetes insipidus.

Compared with patients with GMN, patients in the TIN group were younger and exhibited a shorter duration of pSS (TIN $3.0[1.75,7.50]$ years vs. GMN 11.5 $[2.0,20.0]$ years, $P=0.025)$, and more frequently exhibited elevated IgG levels (TIN $17 / 20$ vs. GMN 8/18, $P=0.016)$. Patients with GMN exhibited more prominent microscopic haematuria (TIN 5/21 vs GMN 13 / $18, P=0.004)$ and proteinuria (TIN $0.8 \pm 0.6 \mathrm{~g} /$ day vs. GMN $3.9 \pm 4.9 \mathrm{~g} /$ day, $P=0.033)$. Renal function at kidney biopsy did not differ between the TIN and GMN groups (Table 1).

Table 1 Demographic, clinical and serological characteristics of patients with pSS-related TIN and GMN

\begin{tabular}{|c|c|c|c|c|}
\hline Items & Total $(n=39)$ & $\operatorname{TIN}(n=21)$ & GMN $(n=18)$ & $P$ \\
\hline Age at KB (years, $M \pm S D$ ) & $42 \pm 14.1$ & $36 \pm 12.5$ & $48 \pm 13.5$ & 0.008 \\
\hline Male:female ratio & $9 / 30$ & $3 / 18$ & $6 / 12$ & NS \\
\hline PSS duration (years, IQR) & $5.0[2.0,16.0]$ & $3.0[1.75,7.50]$ & $11.5[2.0,20.0]$ & 0.025 \\
\hline IS therapy (\%) & 15/39 (38.5) & $8 / 21$ & $7 / 18$ & NS \\
\hline ANA (+) (\%) & 37/39 (94.9) & $21 / 21$ & $16 / 18$ & NS \\
\hline ANA 1:1280 (+) (\%) & $8 / 39(20.5)$ & $5 / 21$ & $3 / 18$ & NS \\
\hline ANA 1:640 (+) (\%) & $16 / 39(41.0)$ & $11 / 21$ & $5 / 18$ & NS \\
\hline Anti-SSA (+) (\%) & $34 / 39(87.2)$ & $18 / 21$ & $16 / 18$ & NS \\
\hline Anti-SSB (+) (\%) & 13/39 (33.3) & $9 / 21$ & $4 / 18$ & NS \\
\hline Serum IgG elevation (\%) & 25/38 (65.8) & $17 / 20$ & $8 / 18$ & 0.016 \\
\hline Serum IgA elevation (\%) & $14 / 38(36.8)$ & $6 / 20$ & $8 / 18$ & NS \\
\hline Serum IgM elevation (\%) & $4 / 38(10.5)$ & $3 / 20$ & $1 / 18$ & NS \\
\hline HypocomplementemiaLow CH50 & $3 / 38(7.9)$ & $0 / 20$ & $3 / 18$ & NS \\
\hline Low C3 & 6/38 (15.8) & $1 / 20$ & $5 / 18$ & NS \\
\hline Low C4 & 6/35 (17.1) & $2 / 17$ & $4 / 18$ & NS \\
\hline RTA(\%) & $22 / 39(56.4)$ & $20 / 21$ & $2 / 18$ & $<0.001$ \\
\hline Urinary calcification/stone (\%) & $12 / 39(30.8)$ & $10 / 21$ & $2 / 18$ & 0.018 \\
\hline Microhaematuria (\%) & $18 / 39(46.2)$ & $5 / 21$ & $13 / 18$ & 0.004 \\
\hline \multicolumn{5}{|l|}{ Proteinuria (\%) } \\
\hline Mild (<1.5 g/day) & 25/39 (64.1) & $17 / 21(81.0)$ & $8 / 18(44.4)$ & \\
\hline Moderate (1.5-3.5 g/day) & 7/39 (17.9) & $4 / 21(19.0)$ & $3 / 18(16.7)$ & \multirow[t]{2}{*}{0.006} \\
\hline Nephrotic range (> 3.5 g/day) & 7/39 (17.9) & $0 / 21(0)$ & 7/18 (38.9) & \\
\hline 24-h urine collection (g/day, $\mathrm{M} \pm \mathrm{SD}$ ) & $2.2 \pm 3.7$ & $0.8 \pm 0.6$ & $3.9 \pm 4.9$ & 0.033 \\
\hline $\operatorname{Scr}(\mu \mathrm{mol} / \mathrm{L}, \mathrm{M} \pm \mathrm{SD})$ & $103.9 \pm 40.99$ & $113.2 \pm 42.33$ & $93.0 \pm 37.60$ & NS \\
\hline eGFR (mL/min.1.73 m², M \pm SD) & $70.2 \pm 27.23$ & $64.0 \pm 28.17$ & $77.5 \pm 24.90$ & NS \\
\hline
\end{tabular}

NS not significant, TIN tubular interstitial nephritis, GMN glomerulonephritis, KB kidney biopsy, IS immunosuppression, $A N A$ anti-nuclear antibodies, RTA renal tubular acidosis, $S c r$ serum creatinine, eGFR estimated glomerular filtration rate (calculated with the CKD-EPI creatinine equation), $M \pm S D$ mean \pm standard deviation, $I Q R$ interquartile range 
Kidney biopsy findings and glomerular C4d deposition Among the 18 patients with GMN, 8 were diagnosed with MN, 5 with MePGN, 2 with IgAN and 3 with MCD. Twenty-one patients were diagnosed with TIN. Specifically, 2 patients were diagnosed with acute interstitial nephritis, and the other 19 were diagnosed with chronic interstitial nephritis.

All 8 patients with $\mathrm{MN}$ exhibited C4d deposition along the glomerular capillary and/ or in mesangium (Fig. 2A1, A2). IF staining revealed IgG deposition in $7 / 8$ of patients with $\mathrm{MN}$ and $\mathrm{C} 3$ deposition in $2 / 8$ of patients with $\mathrm{MN}$. Only 1 patient displayed weak $\mathrm{C} 1 \mathrm{q}$ deposition using IF staining. Sub-epithelial electrondense deposits (EDDs) and occasional mesangial EDDs were also observed in patients with MN using EM, including the patient with negative IgG deposition, confirming the diagnosis of MN (Table 2). Six of the patients with MN were diagnosed with stage II-III disease and exhibited different degrees of mesangial proliferation. Four cases had segmental thickening of GBM. Three out of five patients with MePGN and $1 / 2$ patients with IgAN had mild mesangial deposition of $\mathrm{C} 4 \mathrm{~d}$, but the staining area did not meet the criteria for glomerular $\mathrm{C} 4 \mathrm{~d}$ positive (Fig. 2A3).
Table 2 Details of C4d deposition in patients with pSS-MN

\begin{tabular}{|c|c|c|c|c|c|c|}
\hline \multirow[t]{2}{*}{ Patient } & \multirow{2}{*}{$\begin{array}{l}\text { Scr at KB } \\
(\mu \mathrm{mol} / \mathrm{L})\end{array}$} & \multirow{2}{*}{$\begin{array}{l}\text { Pathological } \\
\text { Diagnosis }\end{array}$} & \multicolumn{3}{|c|}{ C4d } & \multirow[b]{2}{*}{ EDD } \\
\hline & & & G & $\mathrm{TI}$ & PTC & \\
\hline 1 & 74.0 & $\mathrm{MN}$ & + & 1 & 0 & Sub-epi ${ }^{a}$ \\
\hline 2 & 72.0 & $\mathrm{MN}$ & + & 1 & 0 & Sub-epi ${ }^{a}$ \\
\hline 3 & 122.0 & $\mathrm{MN}$ & + & 2 & 2 & NA \\
\hline 4 & 59.0 & $\mathrm{MN}$ & + & 1 & 0 & Sub-epi \\
\hline 5 & 51.0 & $\mathrm{MN}$ & + & 0 & 0 & Sub-epi $i^{a}$ \\
\hline 6 & 95.0 & $\mathrm{MN}$ & + & 2 & 0 & Sub-epi $i^{a}$ \\
\hline 7 & 96.0 & $\mathrm{MN}+\mathrm{TIN}$ & + & 2 & 2 & Sub-epi, mes \\
\hline 8 & 90.0 & $M N+T I N$ & + & 1 & 0 & Sub-epi \\
\hline
\end{tabular}

NA not available, Deposition site: $G$ glomeruli, $T$ T tubulointerstitium, PTC peritubular capillary, EDD electron-dense deposit, mes mesangium, sub-epi sub-epithelium, $M N$ membranous nephropathy, TIN tubular interstitial nephritis, Scr serum creatinine, $K B$ kidney biopsy

${ }^{a}$ in patients with MN, EDDs were mainly observed in the sub-epithelial space and occasionally observed in the mesangium

Tubulointerstitial and peritubular capillary C4d deposition and clinical and pathological differences between groups

Tubulointerstitial C4d deposition was observed in 32 patients $(17 / 21$ patients with TIN and 15/18 patients with GMN), including 16 patients with weak or spotty staining, 12 patients with patchy C4d staining, and 4 patients with diffuse C4d staining (Fig. 2B1, B2 and

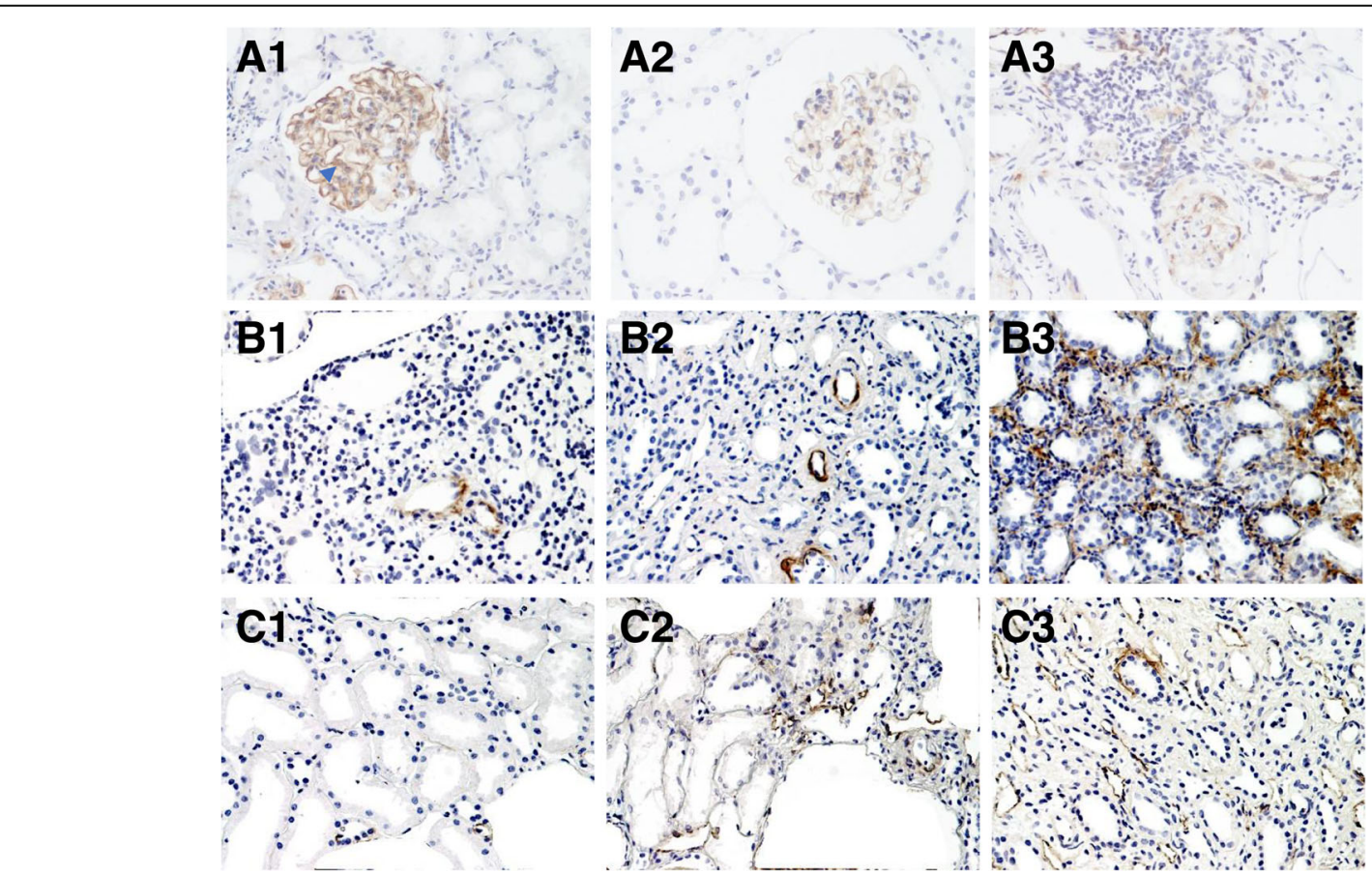

Fig. 2 Renal pathology and C4d deposition in kidney tissues from patients with pSS related renal impairments. pSS renal impairments (light microscopy, IHC). Tubulointerstitial C4d and peritubular capillary C4d staining were semi-quantitatively scored from 1 to 3 . A1 $\times 200$, pSS-related MN, C4d continuous staining along GBM and mild staining in mesangium(arrow). A2 $\times 200$, pSS-related MN, C4d segmental staining along GBM. $\mathbf{A} 3 \times 100$, pSS-related IgAN, mild mesangial staining of $\mathrm{C} 4 \mathrm{~d}$ which did not meet the criteria for $\mathrm{G}-\mathrm{C} 4 \mathrm{~d}+$, mesangial deposition of IgA and $\mathbf{C} 3$ positive. B1 $\times 200$, TI-C4d score 1 . Clinically dRTA, TIN, IF(-). B2×200, TI-C4d score 2. Clinically dRTA, TIN, IF(-). B3 ×200, TI-C4d score 3. Clinically RTA, TIN+mild MePGN, glomerular IF(-), interstitial C3 deposition positive by IF. C1 × 200, PTC C4d score 1 (minimal staining). TIN+MePGN, IF(-). C2 × 200, PTC C4d score 2(focal staining). Early MN, with $\lg G(3+), \lg A(2+)$ and $\mathbf{C 3}(3+)$ deposition along GBM, C1q negative. C3 $\times 200$, PTC-C4d score 3(diffuse staining). TIN 
B3). Two of the 4 patients displaying diffuse tubulointerstitial C4d deposition exhibited tubulointerstitial IgG or C3 deposition. Immune complex deposition was relatively rare in patients with TIN. Four patients with TIN exhibited tubular and interstitial deposition of IgG and C3.

We compared the clinical and pathological characteristics between the tubulointerstitial C4d-positive group (TI-C4d score $>1$ ) and C4d-negative group (Table 3). TI-C4d-positive patients experienced a longer duration of renal involvement $(P=0.001)$ and exhibited increased serum creatinine levels at kidney biopsy $(P=$ 0.008). TI-C4d score had a linear correlation with serum creatinine levels at kidney biopsy $(\beta=17.5 \pm$ 6.92, $P=0.016)$. Pathologically, the TI-C4d ${ }^{+}$group exhibited a higher interstitial fibrosis score $(P=0.035)$ (Table 3). The TI-C4d score exhibited weak correlations with the percentage of sclerotic glomeruli (Spearman's Rho $=0.432, P=0.013$ ), the degree of interstitial infiltration of mononuclear cells $(\mathrm{Rho}=0.339, \mathrm{P}=$ $0.035)$, and the degree of interstitial fibrosis $(\mathrm{Rho}=$ $0.380, P=0.017$ ).

PTC C4d deposition was observed in 12 patients, including 4 patients with minimal deposition (Fig. 2C1), 4 patients with local deposition (Fig. 2C2) and
4 patients with diffuse deposition (Fig. 2C3). The PTC C4d score was positively correlated with the ANA titre (Spearman's Rho $=0.458, P=0.003$ ). All of 4 patients with diffuse deposition had elevation of serum IgG levels, and 3 of them had concomitant elevation of serum IgA levels and ANA titre at 1:1280, the serum levels of IgM were within normal range for all 4 patients.

\section{Treatment and patient follow-up}

Thirty-seven patients received glucocorticoids. Twentyone patients simultaneously received cyclophosphamide therapy. Other immunosuppressive agents included cyclosporine $\mathrm{A}(n=1)$, mycophenolate $\operatorname{mofetil}(\mathrm{n}=1)$, azathioprine $(\mathrm{n}=1)$, methotrexate $(n=6)$, leflunomide(n $=1)$, hydroxychloroquine $(\mathrm{n}=1)$ and tripterygium glycosides $(n=7)$. The median follow-up time was 642 [2391458 ] days. Ten $(25.6 \%)$ patients exhibited a $20 \%$ increase in eGFR at the end of follow-up. The 4 patients with PTC C4d diffuse deposition were treated with prednisone and immunosuppressants (leflunomide in one patient, methotrexate in another and cyclophosphamide in the other two). Two of them were followed for more than half a year and did not exhibit a

Table 3 Comparison between the C4d-positive and C4d-negative groups

\begin{tabular}{|c|c|c|c|}
\hline Items & $\mathrm{TI}^{-C 4 \mathrm{~d}^{+}}(n=16)$ & $\mathrm{TI}^{-C 4 \mathrm{~d}^{-}}(n=23)$ & $P$ \\
\hline Age (years, $\mathrm{M} \pm \mathrm{SD}$ ) & $41 \pm 12.6$ & $42 \pm 15.4$ & NS \\
\hline SS duration (years, $\mathrm{M} \pm \mathrm{SD}$ ) & $9.9 \pm 9.17$ & $8.4 \pm 9.91$ & NS \\
\hline Renal duration (years, IQR) & $4.0[2.0,9.3]$ & $0.9[0.1,2.0]$ & 0.001 \\
\hline ANA positive & $16 / 16$ & $21 / 23$ & NS \\
\hline ANA 1:1280 & $5 / 16$ & $3 / 23$ & NS \\
\hline Anti-SSA (+) & $13 / 16$ & $21 / 23$ & NS \\
\hline Anti-SSB (+) & $6 / 16$ & $7 / 23$ & NS \\
\hline Serum IgG elevation & $11 / 15$ & $14 / 23$ & NS \\
\hline Serum IgA elevation & $8 / 15$ & $6 / 23$ & NS \\
\hline Serum IgM elevation & $3 / 15$ & $1 / 23$ & NS \\
\hline Hypocomplementemia Low CH50 & $2 / 16$ & $1 / 22$ & NS \\
\hline Low C3 & $4 / 16$ & $2 / 22$ & NS \\
\hline Low C4 & $3 / 15$ & $3 / 20$ & NS \\
\hline CRP elevation & $3 / 13$ & $0 / 19$ & 0.058 \\
\hline $\operatorname{Scr}(\mu \mathrm{mol} / \mathrm{L}, \mathrm{IQR})$ & $132.5[89.7,165.5]$ & $83.0[70.7,102.0]$ & 0.008 \\
\hline eGFR (mL/min.1.73 m2, M $\pm S D)$ & $58.7 \pm 27.31$ & $78.3 \pm 24.65$ & 0.025 \\
\hline 24-h urine collection (g/day, $\mathrm{M} \pm \mathrm{SD}$ ) & $1.9 \pm 2.09$ & $2.5 \pm 4.48$ & NS \\
\hline Glomerular sclerosis (\%, IQR) & $19.1[0,49.6]$ & $4.2[1.9,6.3]$ & 0.051 \\
\hline Interstitial fibrosis $(\mathrm{M} \pm \mathrm{SD})$ & $2.3 \pm 1.29$ & $1.4 \pm 1.04$ & 0.035 \\
\hline
\end{tabular}


significant improvement of renal function. In one patient, Scr at the time of kidney biopsy and of the last follow-up time were $164 \mu \mathrm{mol} / \mathrm{L}$ (eGFR $44 \mathrm{~mL} /$ $\min .1 .73 \mathrm{~m}^{2}$ ) and $177 \mu \mathrm{mol} / \mathrm{L}$ (eGFR $40 \mathrm{~mL} / \mathrm{min} .1 .73$ $\mathrm{m}^{2}$ ), respectively. In the other patient, Scr varied from $97 \mu \mathrm{mol} / \mathrm{L}$ (eGFR $57 \mathrm{~mL} / \mathrm{min} .1 .73 \mathrm{~m}^{2}$ ) at the time of kidney biopsy to $86 \mu \mathrm{mol} / \mathrm{L}$ (eGFR $66 \mathrm{~mL} / \mathrm{min} .1 .73 \mathrm{~m}^{2}$ ) at the end of follow-up.

\section{Discussion}

This study investigated the clinical significance of renal C4d deposition in patients with pSS-related renal lesions which has not been reported before. Patients with pSSMN exhibited glomerular C4d deposition without obvious concomitant $\mathrm{C} 1 \mathrm{q}$ deposition, indicating the involvement of the lectin pathway of complement activation in pSS-MN. Tubulointerstitial and PTC C4d deposition were also observed. The tubulointerstitial-C4d score was correlated with kidney function at the time of kidney biopsy, indicating a possible link between renal interstitial injury and autoantibody-mediated complement activation in patients with pSS.

GMN was more prevalent in our study (46.2\%) than in previous studies using serum and urine biochemical examinations (13.9-29\%) [15, 29]. In studies employing pathological investigations, the proportion of GMN ranged from 37.1 to $48.6 \%[7,14,30]$. In the GMN group, there were 8 patients with the pathological diagnosis of MN. The PLA2R/THSD7A antibodies in combination with IgG4 is a useful tool to diagnose intrinsic antibodies related $\mathrm{MN}$ [31].As the retrospective nature of our study, there were not enough biopsy tissues for us to test the expression of PLA2R/THSD7A antibodies as well as IgG subtypes. However, 6 of the 8 patients with $M N$ presented with mesangial proliferation and occasional mesangial EDDs were observed in 5 patients, 4 of them had segmental thickening of GBM, which are more likely features of secondary MN [32]. One patient with negative IF was confirmed by detecting sub-epithelial EDDs under the EM. The IF negativity was possibly due to the early disease stage and lack of abundant immune complex. No obvious clinical and pathological features of lupus nephritis were observed both at the time of kidney biopsy and during follow up, we excluded the possibility of MN secondary to SLE. MN was the most common type of glomerular involvement in our study. In previous studies, MPGN was the most common type of GMN [7,33] and was typically accompanied by cryoglobulinemia. The reason for the increased proportion of patients with MN in our study may be due to differences in environmental and genetic factors, because the Chinese population exhibits an increased prevalence of MN and less prevalence of MPGN [34].

The contribution of complement to MN (primary and secondary) was documented elsewhere [35]. Besides C3, the component of the alternative pathway, glomerular deposition of C4d and MBL were detected in PMN, indicating involvement of the lectin pathway [11, 13]. The lectin pathway of complement may be possibly triggered by the in situ formed immune complexes comprising the IgG4 type of anti-PLA2R antibodies [9]. In patients with SLE-MN, circulating immune complexes deposition and the subsequent activation of the complement system resulted in the full house deposition of immune complexes and $\mathrm{C} 1 \mathrm{q}$, and all the three pathways of complement activation were involved, namely the classical, lectin [22] and alternative pathway [36]. All patients with $\mathrm{MN}$ in our study exhibited glomerular C4d deposition without obvious $\mathrm{C} 1 \mathrm{q}$ deposition, indicating local complement activation by the lectin pathway, which is similar to PMN [37] and different from SLE-MN [38], the underlying mechanism remains to be investigated.

The significance of tubulointerstitial C4d deposition is not clear, and few studies are available. Tubular C4d deposition was associated with an increased grade of WHO classification of IgAN [25]. Patients with TBM C4d deposition exhibited a significantly higher score for the viral cytopathic effect on BK nephropathy [39]. In patients with lupus nephritis, no practical significance of TBM-C4d was identified [40]. We identified spread C4d deposition along TBM and in the renal interstitial compartment with different degree. The semi-quantified TI-C4d score was correlated with worse renal function at kidney biopsy and more severe interstitial fibrosis, suggesting potential contribution of complement activation to tubulointerstitial injuries and renal impairment [41]. For most patients with pSS-related TIN, the IF staining results were negative and cellular immunity is considered a main pathological mechanism. The occasional TBM deposition of complement in images of IF staining is typically considered nonspecific [4]. Technically, immunohistochemistry for TBM C4d in paraffinembedded renal allograft tissues is more specific than IF in frozen tissues ( $4 \%$ by IHC vs. $48 \%$ by IF) [42]. Thus, we considered that the tubulointerstitial C4d deposits identified by IHC in our study were more inclining to suggest its potential role in the pathoetiological aspect. Due to the complexity of the complement system in diseases [43], the cause-effect relationship of complement activation and renal lesions in patients with pSS can not be demonstrated in this study, which certainly is an area needing to be further investigated.

As a marker for antibody-mediated renal allograft rejection [24], PTC C4d has been less frequently reported in glomerulonephritis, and different pathological mechanisms may be involved. In patients with LN, local immune complexes may be responsible for PTC C4d deposition as EDDs along the basement membrane of PTC were identified in 24/31 (77.4\%) of patients with LN presenting PTC 
C4d deposition, whereas none of the patients with acute antibody-mediated rejection exhibited deposition [44]. In addition to autoantibodies, local shear stress on endothelial cells may play a role in the renal crisis in patients with scleroderma [45, 46], and PTC C4d correlated with an increased risk of renal failure [47]. In our study, PTC C4d deposition was identified in 12 patients. The PTC C4d score was positively correlated with the ANA titre. Thus, PTC C4d deposition in pSS-related lesions was possibly mediated by the effects of autoantibodies, which is in accordance with the detection of anti-carbonic anhydrase II antibodies [16] and antibodies against intercalated cells [18] in pSS-related renal tubular lesions. As few patients exhibited diffuse PTC C4d deposition, the significance of PTC C4d deposits in pSS-related renal injury remains to be further clarified.

In consistence with Kidder et al's study showing similar renal survival rate between the GMN and the TIN pathological entities [7], our study also showed that renal function at kidney biopsy in patients with GMN did not differ from those with TIN. PTC C4d deposition was a prognostic marker in renal allograft [48]. Although 10 patients in our study exhibited improvement of renal function, two of the 4 patients with PTC C4d diffuse deposition did not show significant improvement of renal function during follow-up, indicating poor response to treatment. The other two patients with PTC C4d diffuse deposition were lost follow up shortly after discharge, which resulted in the small sample size, the impact of C4d on renal function in patients with pSS related renal impairments remains to be explored in a prospective study with large sample size.

The retrospective nature of this study may lead to possible bias, as more patients with GMN were listed in the kidney biopsy database. Other limitations of our study were that we did not examine the serum anti-PLA2R antibody titre, and an IgG sub-type analysis was not performed in renal tissues. The inadequacy of biopsy tissues hindered the testing of other complement components (such as lectin and C5b-9).

\section{Conclusions}

This preliminary study investigated C4d deposition in patients with pSS with renal involvement. Glomerular C4d positivity was detected in $100 \%$ of patients with pSS-MN, in the absence of $\mathrm{Clq}$, indicating possible complement activation through the lectin pathway. The tubulointerstitial C4d deposition observed in our patients with pSS indicated possible involvement of the MBL pathway of complement activation in pSS-renal lesions. Tubulointerstitial C4d deposits might be a candidate of pathological markers of severe renal injury in patients with pSS-related renal impairments, which remains to be further investigated.

\section{Take Home Messages}

- The MBL pathway of complement activation may be involved in pSS related MN and possibly mediated pSS related tubulointerstitial injury.

\section{Additional file}

Additional file 1: Figure S1. $\times 200$, positive control, C4d stained dendritic cells in the tonsil tissues. Figure $\mathbf{S 2} \times \mathbf{2 0 0}$, negative control, renal biopsy tissues from a patient with minor lesions. (DOCX 604 kb)

\section{Abbreviations}

ANA: Anti-nuclear antibodies; ANCA: Anti-neutrophil cytoplasmic antibodies; CRP: C-reactive protein; EDD: Electron-dense deposit; eGFR: Estimated glomerular filtration rate; EM: Electron microscopy; ESR: Erythrocyte sedimentation rate; FSGS: Focal segmental glomerular sclerosis; GMN: Glomerulonephritis; IF: Immunofluorescence; IgAN: IgA nephropathy; LM: Light microscopy; LN: Lupus nephritis; MBL: Mannosebinding lectin; MCD: Minimal change disease; MePGN: Mesangial proliferative glomerulonephritis; mes: Mesangium; MN: Membranous nephropathy; MPGN: Membranoproliferative glomerulonephritis; PBC: Primary biliary cirrhosis; PLA2R: M-type phospholipase A2 receptor; PMN: Primary membranous nephropathy; PSS: Primary Sjögren's syndrome; PTC: Peritubular capillary; RA: Rheumatoid arthritis; RF: Rheumatoid factor; RTA: Renal tubular acidosis; SLE: Systemic lupus erythematosus; subepi: Sub-epithelium; THSD7A: Anti-thrombospondin type 1 domain containing 7A; TI-C4d: Tubulointerstitial C4d; TIN: Tubulointerstitial nephritis; TMA: Thrombotic microangiopathy

\section{Acknowledgements}

The authors thank Dr. Jianling Tao (Department of Medicine, Stanford University) for editing the manuscript.

\section{Funding}

This work was supported by the Peking Union Medical College Hospital Science Fund for Junior Faculty [grant number 20132755]. It is used according to the budget plan. Briefly, this funding was used mainly in the acquisition of laboratory materials, analysis, and interpretation of data.

Availability of data and materials

Not applicable.

\section{Authors' contributions}

WX participated in the study design, obtained clinical data, performed the immunohistochemical staining, performed the statistical analysis, and wrote the manuscript. BG helped obtain the clinical data, participated in performing the statistical analyses and helped with drafting the manuscript. LD and YL participated in the immunohistochemical staining. WY participated in the pathological diagnosis and in the data analysis. LCh and XL participated in study design and coordination and helped with the acquisition of clinical data. ALL critically revised the manuscript for important intellectual content. FZh participated in the study design and coordination and helped to conceive the study. ML conceived the study, participated in its design and coordination, and helped to draft the manuscript and critically revise the manuscript. All authors read and approved the final manuscript.

\section{Ethics approval and consent to participate}

This study was performed in accordance with the Declaration of Helsinki. Written informed consent was obtained from every patient being analyzed in this study before kidney biopsy, which was clearly addressed that "the left kidney biopsy sample and the relevant clinical information could be used retrospectively in clinical study". This study was approved by the Ethics Committee of Peking Union Medical College Hospital (Reference code:S-k585), where waiver of consent for this retrospective clinical study was approved. 


\section{Competing interests}

The authors declare that they have no competing interests.

\section{Publisher's Note}

Springer Nature remains neutral with regard to jurisdictional claims in published maps and institutional affiliations.

\section{Author details}

'Department of Nephrology, Peking Union Medical College Hospital, Chinese Academy of Medical Sciences, Peking Union Medical College, No. 1 Shuaifuyuan, Wangfujing Street, Beijing 100730, China. ${ }^{2}$ Department of Nephrology, Beijing Jishuitan Hospital, Fourth Clinical College, Peking University, Beijing 100035, China. ${ }^{3}$ Renal Division, Department of Medicine, Institute of Nephrology, Key Laboratory of Renal Disease, Ministry of Health of China, Peking University First Hospital, Peking University, Beijing 100034, China.

Received: 28 November 2018 Accepted: 17 April 2019 Published online: 28 May 2019

\section{References}

1. Ramos-Casals M, Brito-Zeron P, Solans R, Camps MT, Casanovas A, Sopena B, et al. Systemic involvement in primary Sjogren's syndrome evaluated by the EULAR-SS disease activity index: analysis of 921 Spanish patients (GEAS-SS registry). Rheumatology (Oxford). 2014:53:321-31.

2. Garcia-Carrasco M, Ramos-Casals M, Rosas J, Pallares L, Calvo-Alen J, Cervera $R$, et al. Primary Sjogren syndrome: clinical and immunologic disease patterns in a cohort of 400 patients. Medicine. 2002;81:270-80.

3. Francois $H$, Mariette $X$. Renal involvement in primary Sjogren syndrome. Nat Rev Nephrol. 2016;12:82-93.

4. Evans RD, Laing CM, Ciurtin C, Walsh SB. Tubulointerstitial nephritis in primary Sjogren syndrome: clinical manifestations and response to treatment. BMC Musculoskelet Disord. 2016;17:2.

5. Jasiek M, Karras A, Le Guern V, Krastinova E, Mesbah R, Faguer S, et al. A multicentre study of 95 biopsy-proven cases of renal disease in primary Sjogren's syndrome. Rheumatology (Oxford). 2017;56:362-70.

6. Maripuri S, Grande JP, Osborn TG, Fervenza FC, Matteson EL, Donadio JV, et al. Renal involvement in primary Sjögren's syndrome: a clinicopathologic study. Clin J Am Soc Nephrol. 2009;4:1423-31.

7. Kidder D, Rutherford E, Kipgen D, Fleming S, Geddes C, Stewart GA. Kidney biopsy findings in primary Sjogren syndrome. Nephrol Dial Transplant. 2015;30:1363-9.

8. Carrillo-Pérez DL, Tejeda-Maldonado J, Garza-García C, Soto-Abraham V, Hernández-Molina G, Molina-Paredes GA, et al. Biopsy-proven renal involvement and prognosis in 13 hispanic patients with primary Sjögren syndrome. Med Clín (English Edition). 2018;150:43-8.

9. Sinico RA, Mezzina N, Trezzi B, Ghiggeri GM, Radice A. Immunology of membranous nephropathy: from animal models to humans. Clin Exp Immunol. 2016;183:157-65

10. Beck LH Jr, Bonegio RG, Lambeau G, Beck DM, Powell DW, Cummins TD, et al. M-type phospholipase A2 receptor as target antigen in idiopathic membranous nephropathy. N Engl J Med. 2009;361:11-21.

11. Hayashi N, Okada K, Matsui Y, Fujimoto K, Adachi H, Yamaya H, et al. Glomerular mannose-binding lectin deposition in intrinsic antigen-related membranous nephropathy. Nephrol Dial Transplant. 2018;33:832-40.

12. Tomas NM, Beck LH Jr, Meyer-Schwesinger C, Seitz-Polski B, Ma H, Zahner G, et al. Thrombospondin type-1 domain-containing 7A in idiopathic membranous nephropathy. N Engl J Med. 2014;371:2277-87.

13. Hui M, Uppin MS, Prayaga AK, Raju SB, Rajasekhar L. C4d immunohistochemistry in membranous nephropathy. J Lab Physicians. 2014;6:76-9.

14. Goules AV, Tatouli IP, Moutsopoulos HM, Tzioufas AG. Clinically significant renal involvement in primary Sjogren's syndrome: clinical presentation and outcome. Arthritis Rheum. 2013;65:2945-53.

15. Bossini N, Savoldi S, Franceschini F, Mombelloni S, Baronio M, Cavazzana I, et al. Clinical and morphological features of kidney involvement in primary Sjögren's syndrome. Nephrol Dial Transplant. 2001;16:2328-36.

16. Takemoto F, Hoshino J, Sawa N, Tamura Y, Tagami T, Yokota M, et al. Autoantibodies against carbonic anhydrase II are increased in renal tubular acidosis associated with Sjögren syndrome. Am J Med. 2005;118:181-4.

17. Takemoto F, Katori H, Sawa N, Hoshino J, Suwabe T, Sogawa Y, et al. Induction of anti-carbonic-anhydrase-II antibody causes renal tubular acidosis in a mouse model of Sjögren's syndrome. Nephron Physiol. 2007; 106:p63-p8.

18. Devuyst O, Lemaire M, Mohebbi N, Wagner CA. Autoantibodies against intercalated cells in Sjogren's syndrome. Kidney Int. 2009;76:229.

19. Ehrnthaller C, Ignatius A, Gebhard F, Huber-Lang M. New insights of an old defense system: structure, function, and clinical relevance of the complement system. Mol Med. 2011;17:317-29.

20. Sethi S, Nasr SH, De Vriese AS, Fervenza FC. C4d as a diagnostic tool in proliferative GN. J Am Soc Nephrol. 2015;26:2852-9.

21. Espinosa M, Ortega R, Gómez-Carrasco JM, López-Rubio F, López-Andreu M, López-Oliva MO, et al. Mesangial C4d deposition: a new prognostic factor in IgA nephropathy. Nephrol Dial Transplant. 2009;24:886-91.

22. Kim MK, Maeng YI, Lee SJ, Lee IH, Bae J, Kang YN, et al. Pathogenesis and significance of glomerular C4d deposition in lupus nephritis: activation of classical and lectin pathways. Int J Clin Exp Pathol. 2013;6:2157-67.

23. Chua JS, Baelde HJ, Zandbergen M, Wilhelmus S, van Es LA, de Fijter JW, et al. Complement factor C $4 \mathrm{~d}$ is a common denominator in thrombotic Microangiopathy. J Am Soc Nephrol. 2015;26:2239-47.

24. Regele H, Bohmig GA, Habicht A, Gollowitzer D, Schillinger M, Rockenschaub S, et al. Capillary deposition of complement split product C4d in renal allografts is associated with basement membrane injury in peritubular and glomerular capillaries: a contribution of humoral immunity to chronic allograft rejection. J Am Soc Nephrol. 2002;13:2371-80.

25. Maeng Y-I, Kim M-K, Park J-B, Cho C-H, Oh H-K, Sung WJ, et al. Glomerular and tubular C4d depositions in IgA nephropathy: relations with histopathology and with albuminuria. Int J Clin Exp Pathol. 2013;6:904-10.

26. Zwirner J, Felber E, Schmidt P, Riethmuller G, Feucht HE. Complement activation in human lymphoid germinal centres. Immunology. 1989;66:270-7.

27. Tan Y, Yu F, Qu Z, Su T, Xing GQ, Wu LH, et al. Modified C-reactive protein might be a target autoantigen of TINU syndrome. Clin J Am Soc Nephrol. 2011;6:93-100.

28. Haas M, Sis B, Racusen LC, Solez K, Glotz D, Colvin RB, et al. Banff 2013 meeting report: inclusion of $c 4 d$-negative antibody-mediated rejection and antibody-associated arterial lesions. Am J Transplant. 2014;14:272-83.

29. Ren H, Wang W-M, Chen X-N, Zhang W, Pan X-X, Wang X-L, et al. Renal involvement and followup of 130 patients with primary Sjögren's syndrome. J Rheumatol. 2008:35:278-84.

30. Kaufman I, Schwartz D, Caspi D, Paran D. Sjögren's syndrome-not just Sicca: renal involvement in Sjögren's syndrome. Scand J Rheumatol. 2008;37:213-8.

31. L'Imperio V, Pieruzzi F, Sinico RA, Nebuloni M, Granata A, Smith A, et al. Routine immunohistochemical staining in membranous nephropathy: in situ detection of phospholipase A2 receptor and thrombospondin type 1 containing 7A domain. J Nephrol. 2018;31:543-50.

32. Larsen CP, Messias NC, Silva FG, Messias E, Walker PD. Determination of primary versus secondary membranous glomerulopathy utilizing phospholipase A2 receptor staining in renal biopsies. Mod Pathol. 2013;26:709-15.

33. Matignon M, Cacoub P, Colombat M, Saadoun D, Brocheriou I, Mougenot B, et al. Clinical and morphologic spectrum of renal involvement in patients with mixed cryoglobulinemia without evidence of hepatitis C virus infection. Medicine. 2009;88:341-8.

34. Pan $X, X u J$, Ren $H$, Zhang $W, X u Y$, Shen $P$, et al. Changing spectrum of biopsy-proven primary glomerular diseases over the past 15 years: a singlecenter study in China. Contrib Nephrol. 2013;181:22-30.

35. Ma H, Sandor DG, Beck LH Jr. The role of complement in membranous nephropathy. Semin Nephrol. 2013;33:531-42.

36. Song D, Guo WY, Wang FM, Li YZ, Song Y, Yu F, et al. Complement alternative pathways activation in patients with lupus nephritis. Am J Med Sci. 2017;353:247-57.

37. Espinosa-Hernández M, Ortega-Salas R, López-Andreu M, Gómez-Carrasco JM, Pérez-Sáez MJ, Pérez-Seoane C, et al. C4d as a diagnostic tool in membranous nephropathy. Nefrologia. 2012;32:295-9.

38. Kim SH, Jeong HJ. Glomerular C4d deposition indicates in situ classic complement pathway activation, but is not a marker for lupus nephritis activity. Yonsei Med J. 2003:44:75-80.

39. Batal I, Zainah H, Stockhausen S, Basu A, Tan H, Shapiro R, et al. The significance of renal C4d staining in patients with BK viruria, viremia, and nephropathy. Mod Pathol. 2009;22:1468-76.

40. Batal I, Liang K, Bastacky S, Kiss L, McHale T, Wilson N, et al. Prospective assessment of $\mathrm{C} 4 \mathrm{~d}$ deposits on circulating cells and renal tissues in lupus nephritis: a pilot study. Lupus. 2012;21:13-26.

41. Abbate M, Zoja C, Corna D, Rottoli D, Zanchi C, Azzollini N, et al. Complement-mediated dysfunction of glomerular filtration barrier accelerates progressive renal injury. J Am Soc Nephrol. 2008;19:1158-67. 
42. Batal I, Girnita A, Zeevi A, Saab BA, Stockhausen S, Shapiro R, et al. Clinical significance of the distribution of C4d deposits in different anatomic compartments of the allograft kidney. Mod Pathol. 2008;21:1490-8.

43. Ricklin D, Reis ES, Lambris JD. Complement in disease: a defence system turning offensive. Nat Rev Nephrol. 2016;12:383-401.

44. Li SJ, Liu ZH, Zen CH, Wang QW, Wang Y, Li LS. Peritubular capillary C4d deposition in lupus nephritis different from antibody-mediated renal rejection. Lupus. 2007;16:875-80.

45. Okroj M, Johansson M, Saxne T, Blom AM, Hesselstrand R. Analysis of complement biomarkers in systemic sclerosis indicates a distinct pattern in scleroderma renal crisis. Arthritis Res Ther. 2016;18:267.

46. Yin W, Ghebrehiwet B, Weksler B, Peerschke El. Regulated complement deposition on the surface of human endothelial cells: effect of tobacco smoke and shear stress. Thromb Res. 2008;122:221-8.

47. Batal I, Domsic RT, Shafer A, Medsger TA Jr, Kiss LP, Randhawa P, et al. Renal biopsy findings predicting outcome in scleroderma renal crisis. Hum Pathol. 2009:40:332-40.

48. David-Neto E, Prado E, Beutel A, Ventura CG, Siqueira SA, Hung J, et al. C4dpositive chronic rejection: a frequent entity with a poor outcome. Transplantation. 2007:84:1391-8.

Ready to submit your research? Choose BMC and benefit from:

- fast, convenient online submission

- thorough peer review by experienced researchers in your field

- rapid publication on acceptance

- support for research data, including large and complex data types

- gold Open Access which fosters wider collaboration and increased citations

- maximum visibility for your research: over $100 \mathrm{M}$ website views per year

At $\mathrm{BMC}$, research is always in progress.

Learn more biomedcentral.com/submissions 